\title{
Allergic bronchopulmonary fungal disease without clinical asthma
}

\author{
JOHN J GLANCY, JANET L ELDER, AND ROSE MCALEER
}

From the Departments of Radiology and Respiratory Medicine, Sir Charles Gairdner Hospital, and State Health Laboratories, Medical Mycology Division, Perth, Western Australia

ABSTRACT During a retrospective survey of patients with pulmonary shadows and blood eosinophilia between the years 1965 and 1980, 42 patients were found with allergic bronchopulmonary fungal disease. Eleven of these had no clinical evidence of asthma. Three of these 11 had hypersensitivity to fungi other than Aspergillus sp. In the absence of asthma there was some difficulty in making a diagnosis, particularly where collapse of the upper lobe occurred in middle-aged or elderly patients and bronchogenic carcinoma was presumed responsible. We suggest that the term allergic bronchopulmonary aspergillosis be replaced by allergic bronchopulmonary fungal disease, the diagnosis be considered in patients with lung disease and blood eosinophilia even in the absence of asthma, and a wider range of fungal allergens be used for skin and precipitin tests.

The increasing frequency with which the diagnosis of allergic bronchopulmonary aspergillosis is being made is probably the result of increasing recognition of the disease entity, ${ }^{1-3}$ and perhaps some liberalisation of the criteria required for diagnosis. The list of criteria usually required begins with asthma and includes blood eosinophilia and transient pulmonary infiltrates. Immediate positive skin tests to Aspergillus $s p$, precipitating antibody against Aspergillus $s p$, elevated IgE concentrations, and where possible, proximal bronchiectasis are required in the USA. ${ }^{13-5}$ British criteria require the presence of asthma, blood eosinophilia, transient infiltrates, and a positive prick test to the fungus. ${ }^{2}$ 6-9 Previous reports have, however, included isolated instances in which the patient did not have asthma. 5810

Thirty-one cases of allergic bronchopulmonary aspergillosis diagnosed by the above criteria have been seen at Sir Charles Gairdner Hospital. Eleven additional patients conforming to the above criteria but with no history of asthma as defined clinically by episodes of wheezing or dyspnoea, and no clinical evidence of airflow obstruction at the time of diagnosis form the basis of this paper.

\section{Methods}

During a retrospective survey of patients with lung opacities and blood eosinophilia seen at the Sir

Address for reprint requests. Dr John J Glancy, Sir Charles Gairdner Hospital, Verdun Street, Nedlands, Western Australia 6009.
Charles Gairdner Hospital between 1965 and 1980, 31 cases of allergic bronchopulmonary aspergillosis conforming to the usual criteria were found. In addition there were 11 patients with similar findings but with no history of asthma - that is, no history of wheezing or dyspnoea and no clinical evidence of airflow obstruction at the time of the diagnosis.

Criteria for diagnosis centred mainly on a positive prick test ${ }^{11}$ to extracts of Aspergillus fumigatus or $A$ terreus and in two patients to an extract of the fungus Helminthosporium sp. A wheal greater than $3 \mathrm{~mm}$ within 15 minutes was considered positive. Atopy or an immediate type 1 sensitivity was determined by means of prick tests to 19 common allergens using Commonwealth Serum Laboratories antigen. Total IgE estimation was performed in eight but specific IgE by means of a RAST test in only three patients. Blood was examined for eosinophils and serum was examined for precipitins to Aspergillus by Ouchterlony agar gel double diffusion in all patients.

Early morning sputa, collected after first rinsing out the mouth with water, were examined by the mycology laboratory for mycelium and were cultured on appropriate media at both $26^{\circ} \mathrm{C}$ and $37^{\circ} \mathrm{C}$.

All patients had chest radiography on several occasions. All patients had ventilatory function tests on more than one occasion, and eight had ventilatory function tests performed before and after the inhalation of two puffs of sympathomimetic bronchodilator aerosol. Two patients had lobectomies and one a segmental resection before a diagnosis was made. 


\section{Results}

All 11 patients, whose ages ranged from 15 to 65 years, denied a history of asthma and none had clinical evidence of generalised airway obstruction at the time of diagnosis. Five admitted to a previous history of rhinitis or hayfever. Eight patients had ventilatory function tests before and after bronchodilators. Six showed a change of less than $9 \%$. Case 10 exhibited a change of $17 \%$ and case $9,34 \%$ (table 1).

Eight patients had a positive prick test to Aspergillus and two to Helminthosporium. Three had a negative prick skin test to all 19 antigens and in one the test was equivocal. Of these three the total $\mathrm{IgE}$ was normal in only one (case 2). A summary of results is given in table 2 .

All patients had a raised blood eosinophil count which ranged from $8 \%$ of $8900 \mathrm{WBC}\left(801 \mathrm{~mm}^{3}\right)$ to $34 \%$ of $13700\left(4685 \mathrm{~mm}^{3}\right)$. Eight patients had an excess of eosinophils in the sputum and one other had Curshman's spirals.

Mycological investigations on specimens from these patients gave the following results. Cases 1,4 , and 9 had septate branching mycelium in the sputum and Aspergillus fumigatus was cultured. Their serum contained antibody to $A$ fumigatus. Case 3 , also with mycelium in the sputum, grew various combinations of $A$ terreus, $A$ fumigatus, $A$ niger, and $A$ flavus. Precipitins were positive to all four species but were strongly positive for $A$ terreus and $A$ fumigatus which each showed four bands. Cases 2 and 5 , in whose sputum no mycelium was found, grew $A$ fumigatus from at least two sputum specimens and had positive precipitins for $A$ fumigatus. One patient (case 7) had masses of septate branching mycelium similar to that of Aspergillus in a plug from a bronchoscopy specimen but the fungus failed to grow. Precipitins were positive for $A$ fumigatus. Cases 8, 10, and 11 all had mycelium on microscopy of sputum. Case 8 grew $D$ hawaiiensis from six sputum samples. This fungus is closely related to and cross reacts with Helminthosporium. Case 10 grew $C$ lunata from five sputa and the resected specimen. Case 11 grew both $D$ hawaiiensis and $C$ lunata from several specimens. Precipitins to aspergilli were negative in all three cases but positive to both $D$ hawaiiensis and $C$ lunata in case 11. Suitable extracts of these fungi were not available at the time of diagnosis of cases 8 and 10. These latter three cases are discussed in the accompanying paper. ${ }^{12}$

The radiological characteristics of this series of patients did not vary significantly from previously reported series, ${ }^{8} 1314$ but perhaps varied quantitatively. Five had lobar collapse at one time or another and one had lobar consolidation. Three had classical branching bronchoceles in the upper lobes and two in the right lower lobe. Fleeting opacities were seen at some time in only six of these patients and on some occasions occurred after the diagnosis had been made. Bronchial wall thickening and bronchiectasis were present on the plain films of several patients. Six patients had bronchograms but only two exhibited classical central bronchiectasis. One other with a bronchocele showed no filling of the segmental bronchus to that area. In the other, contrast outlined the head of the mucus plug in the proximal portion of the segmental bronchus.

\section{Discussion}

Hinson et al $^{6}$ stated that Renon in 1879 first associated Aspergillus $s p$ with asthma. Asthma is the first criterion for a diagnosis of allergic bronchopulmonary aspergillosis in all reported series. However some important series do include occasional cases who did not have clinical asthma. ${ }^{5810}$ It is difficult to determine the exact number but a thorough search of the literature indicates that approximately 10 patients with allergic bronchopulmonary aspergillosis without asthma have previously been reported.

In 42 patients in whom a diagnosis of allergic bronchopulmonary fungal disease was made there were 11 who conformed to the accepted criteria for

Table 1 Pulmonary function tests before and after bronchodilator inhalation

\begin{tabular}{|c|c|c|c|c|c|c|}
\hline \multirow[t]{2}{*}{ Case } & \multicolumn{3}{|c|}{ Vital capacity } & \multicolumn{3}{|l|}{$F E V_{1}$} \\
\hline & Before & After & Predicted & Before & After & Predicted \\
\hline 1 & $3 \cdot 16$ & Not done & 3.61 & $1 \cdot 82$ & Not done & $2 \cdot 58$ \\
\hline 2 & $2 \cdot 77$ & $2 \cdot 58$ & $2 \cdot 49$ & $1 \cdot 88$ & $1 \cdot 60$ & 1.94 \\
\hline 3 & $1 \cdot 79$ & $1 \cdot 79$ & $2 \cdot 30$ & $1 \cdot 37$ & $1 \cdot 40$ & $2 \cdot 00$ \\
\hline 4 & $2 \cdot 87$ & Not done & $2 \cdot 70$ & $1 \cdot 81$ & Not done & $2 \cdot 15$ \\
\hline 5 & $4 \cdot 87$ & $4 \cdot 98$ & $5 \cdot 12$ & $3 \cdot 40$ & $3 \cdot 61$ & $4 \cdot 24$ \\
\hline 6 & $3 \cdot 29$ & Not done & $4 \cdot 40$ & $2 \cdot 63$ & Not done & $3 \cdot 39$ \\
\hline 7 & $2 \cdot 65$ & $2 \cdot 77$ & $4 \cdot 50$ & $1 \cdot 55$ & $1 \cdot 68$ & $3 \cdot 29$ \\
\hline 8 & $3 \cdot 10$ & $3 \cdot 00$ & $3 \cdot 20$ & $2 \cdot 70$ & $2 \cdot 65$ & $2 \cdot 70$ \\
\hline 9 & $1 \cdot 70$ & $2 \cdot 12$ & $2 \cdot 25$ & 0.98 & $1 \cdot 32$ & $1 \cdot 85$ \\
\hline 10 & $3 \cdot 68$ & $3 \cdot 78$ & $3 \cdot 36$ & $2 \cdot 34$ & $2 \cdot 75$ & $2 \cdot 80$ \\
\hline 11 & $6 \cdot 20$ & $6 \cdot 30$ & $5 \cdot 6$ & $4 \cdot 32$ & $4 \cdot 56$ & $4 \cdot 4$ \\
\hline
\end{tabular}




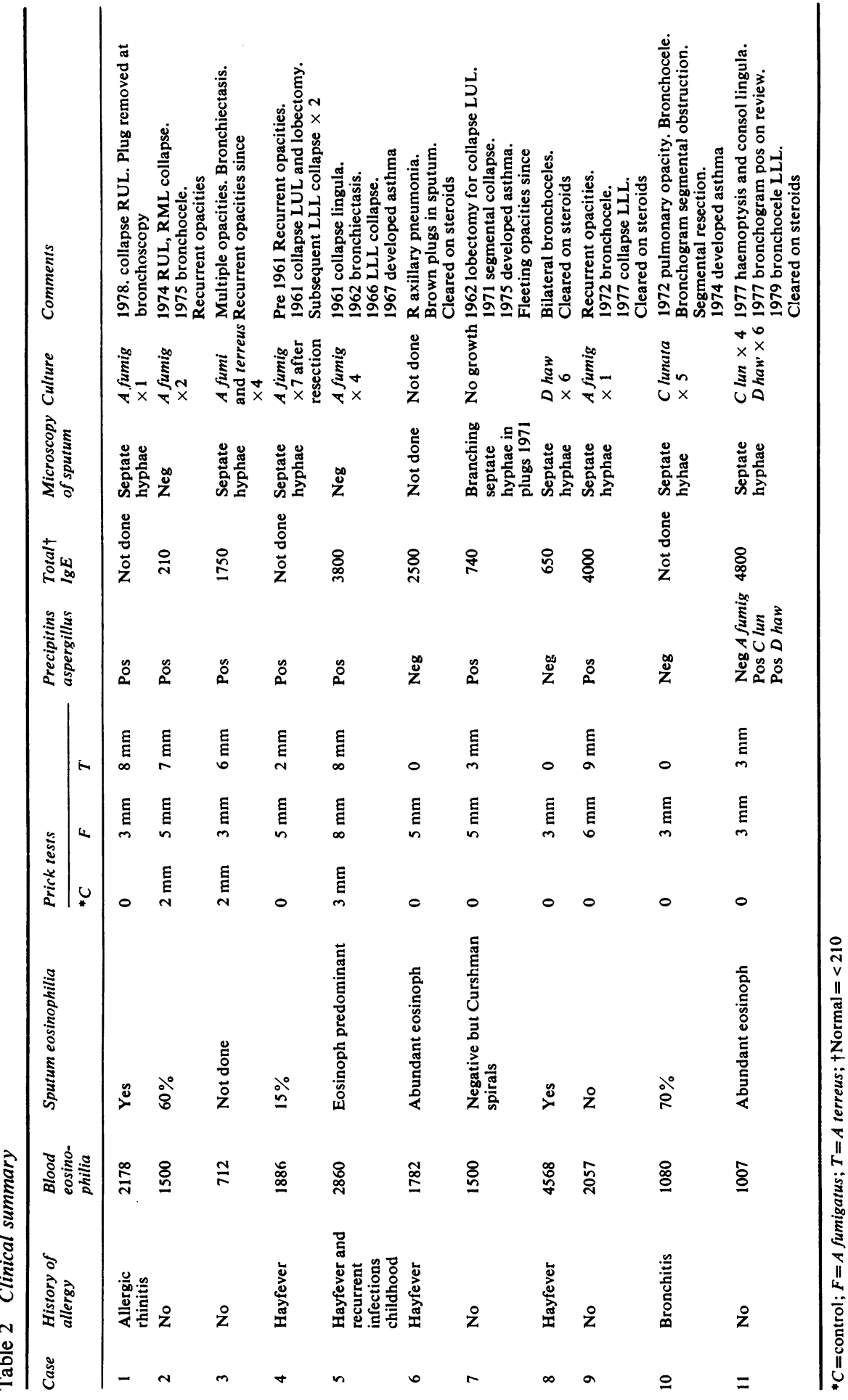


diagnosis except for the absence of clinical asthma. Their ages ranged from 15-65 years. This suggests that in $26 \%$ of patients with this syndrome the diagnosis will be missed if clinical asthma is considered essential for diagnosis.

Three patients developed asthma years after diagnosis-case 510 years; case 7 four years, and case 10 two years after. Steroids used for the treatment of the syndrome may have masked the development of asthma in cases $1,3,4$, and 8 but case 2 has only had occasional brief courses of steroids when opacities have appeared on chest radiographs, and has not developed asthma in the four years since diagnosis. Case 11 was first seen four years ago with haemoptysis and lingular consolidation. He was undiagnosed, untreated, and non-asthmatic even on laboratory testing. He is now on steroids. Case 6 has been lost to follow-up. Case 9 was a heavy smoker with a history of occasional episodes of bronchitis. She was not considered to have asthma by her general practitioner or a physician she had attended for some years. Pulmonary function tests were performed after recurrent opacities on serial chest radiographs suggested the diagnosis of allergic bronchopulmonary fungal disease. These tests showed reversible obstruction diagnostic of asthma (table 1). Case 10 was considered to have recurrent bronchitis after measles pneumonia at the age of 12 years and was treated with antibiotics. On admission to this hospital at 32 years of age she had no clinical evidence of asthma but she did have wheezes localised to the right chest and her $\mathrm{FEV}_{1}$ improved by $17 \%$ after bronchodilator, which is suggestive but not diagnostic of asthma. A bronchogram showed obstruction to the superior segment of the right lower lobe. Two years after surgery she developed clinical asthma.

Difficulty in recognising the nature of the disease process in the absence of asthma did occur in some patients. Cases 4 and 7 had an upper lobectomy and case 10 a segmental resection before diagnosis. A fourth patient (case 1) had collapse of the right upper lobe with a presumptive diagnosis of carcinoma; a preoperative bronchoscopy revealed an obstructing mucous plug. Case 3, a boy of 15 years, had previously been considered to have cystic fibrosis, although sweat electrolyte concentrations were not elevated. Despite a positive bronchogram four years ago, case 11 remained undiagnosed and untreated because of the absence of asthma even on laboratory testing.

Three patients had fungi other than Aspergillus as a cause of their pulmonary disease, case 8 Drechslera hawaiiensis, case 10 Curvularia lunata, and case 11 both of these fungi. In view of these three cases in which fungi other than Aspergillus have caused this syndrome and a previous report ${ }^{15}$ of two similar patients of bronchopulmonary Helminthosporium and the possible incrimination of Candida by Pepys, ${ }^{16}$ we suggest that the more usual term, allergic bronchopulmonary aspergillosis be widened to allergic bronchopulmonary fungal disease. In cases of mucoid impaction with negative tests to Aspergillus or cryptogenic pulmonary eosinophilia a wider range of fungal allergens should be used for skin and precipitin testing.

\section{Addendum}

Since this paper was accepted for publication, a single case of allergic bronchopulmonary fungal disease with asthma caused by Stemphylium lanuginosum has been reported from South Africa. ${ }^{17}$

We would like to thank the Chest Physicians of the Sir Charles Gairdner Hospital for access to these cases and Mrs Leah Bateson for secretarial assistance with multiple drafts of the manuscript.

\section{References}

1 Slavin RG, Stanczyk DJ, Lonigro AJ, Broun GO. Allergic bronchopulmonary aspergillosis-a North American rarity. Clinical and immunologic characteristics. Am J Med 1969; 47:306-13.

2 Editorial. Diagnosing allergic bronchopulmonary aspergillosis. Br Med J 1977; 2:1439-40.

3 Hoehne J, Reed C, Dickie H. Allergic bronchopulmonary aspergillosis is not rare. $\mathrm{J}$ Lab Clin Med 1971; 78:1007-8.

4 Khan ZU, Sandhu RS, Randhawa HS, Menon MPS, Dusa JIS. Allergic bronchopulmonary aspergillosis: a study of 46 cases with special reference to laboratory aspects. Scand J Respir Dis 1976; 57:73-87.

5 Rosenberg M, Patterson R, Mintzer R, Cooper BJ, Roberts M, Harris KE. Clinical and immunologic criteria for the diagnosis of allergic bronchopulmonary aspergillosis. Ann Intern Med 1977; 86:405-14.

6 Hinson KFW, Moon AJ, Plummer NS. Bronchopulmonary aspergillosis: a review and a report of eight new cases. Thorax 1952; 7:317-33.

7 Henderson AH. Allergic aspergillosis: review of 32 cases. Thorax 1968; 23:501-12.

8 McCarthy DS, Simon G, Hargreave FE. The radiological appearances in allergic bronchopulmonary aspergillosis. Clin Radiol 1970; 21:366-75.

9 Malo JL, Hawkins R, Pepys J. Studies in chronic allergic bronchopulmonary aspergillosis. 1 . Clinical and physiological findings. Thorax 1977; 32:254-61.

10 Elder JL, Smyth JT. Allergic bronchopulmonary aspergillosis. Med J Aust 1967; 1:231-2.

11 Longbottom JL, Pepys J. Pulmonary aspergillosis: diagnostic and immunological significance of antigens of c-substances in Aspergillus fumigatus. J Path Bact $1964 ; 88: 141-51$.

12 McAleer R, Kroenert DB, Elder JL, Froudist JH. Allergic bronchopulmonary disease caused by 
Curvalaria lunata and Drechslera hawaiiensis. Thorax. $1981 ; 36: 338-44$.

13 Malo JL, Pepys J, Simon G. Studies in chronic allergic bronchopulmonary aspergillosis. 2. Radiological findings. Thorax 1977; 32:262-8.

14 Mintzer RA, Rogers LF, Kruglik GD, Rosenberg M, Neiman HL, Patterson R. The spectrum of radiologic findings in allergic bronchopulmonary aspergillosis. Radiology 1978; 127:301-7.
15 Dolan CT, Weed LA, Dines DE. Bronchopulmonary helminthosporiosis. Am J Clin Path 1970; 53:235-42.

16 Pepys J. Hypersensitivity disease of the lungs due to fungi and organic dusts. Monogr Allergy 1969; 4:63-8.

17 Benatar SR, Allan B, Hewitson RP, Dou PA. Allergic bronchopulmonary stemphyliosis. Thorax $1980 ; 35: 515-8$. 